\title{
Transfusion Independence Achieved with Combination Fedratinib and Luspatercept in an Elderly Man with Heavily Pretreated Intermediate-2 Risk Primary Myelofibrosis
}

\author{
Nicole K. Yun ${ }^{a}$ Taha Alrifai $^{\mathrm{b}}$ Ira J. Miller ${ }^{\mathrm{c}}$ Jamile M. Shammo ${ }^{\mathrm{b}}$ \\ aDepartment of Internal Medicine, Rush University Medical Center, Chicago, IL, USA; \\ bDivision of Hematology, Oncology, and Cell Therapy, Rush University Medical Center, \\ Chicago, IL, USA; 'Department of Pathology, Rush University Medical Center, Chicago, IL, \\ USA
}

\section{Keywords}

Myeloproliferative neoplasm · Primary myelofibrosis · Fedratinib · Luspatercept · Transfusion independence $\cdot$ Heavily pretreated

\begin{abstract}
Myelofibrosis (MF)-associated anemia and transfusion dependency are associated with inferior quality of life and poor prognosis. JAK2 inhibitors and TGF- $\beta$ superfamily ligand traps are being explored as treatment options for MF-associated anemia. Here, we present the case of a 66-year-old man with heavily pretreated intermediate-2 (INT-2) risk primary MF who had an exceptional response to combination fedratinib and luspatercept therapy. He achieved transfusion independence and experienced a reduction in spleen size from $20 \mathrm{~cm}$ to $12 \mathrm{~cm}$, with remarkable improvement in performance status. Compared with other JAK inhibitors, the mechanism of action of fedratinib may explain its milder effect on anemia. It is possible that the addition of luspatercept may result in an additive or synergistic effect of one or both medications. Although the exact biological pathways have not yet been elucidated, combination fedratinib and luspatercept nevertheless is a promising therapy for anemia in patients with transfusion-dependent INT-2 risk MF.
\end{abstract}




\section{Introduction}

Myelofibrosis (MF) is a $B C R-A B L 1$-negative myeloproliferative neoplasm that is often accompanied by JAK2, CALR, or MPL mutations. The disease manifests clinically as anemia, hepatosplenomegaly, cachexia, and constitutional symptoms such as fatigue, night sweats, and fever [1]. It can occur de novo (primary MF [PMF]) or secondary to polycythemia vera or essential thrombocythemia (secondary MF) [2]. Treatment decisions are based upon symptom burden and risk stratification using variations of the International Prognostic Scoring System (IPSS) rather than disease subtype or molecular profile [3, 4]. Observation alone is typically recommended for low-risk disease. Unfortunately, for intermediate or high-risk MF, there is no therapy which has been clearly proven to have a disease-modifying effect and allogeneic stem cell transplantation remains the only curative therapy in those eligible patients who have high- or intermediate-2 (INT-2) risk disease [5]. The major therapeutic challenge for patients with intermediate to high-risk MF is to improve anemia, decrease the need for supportive packed red blood cell transfusions, mitigate toxicity of iron overload, and ultimately, to enhance quality of life. We describe the case of an elderly patient with heavily pretreated INT-2 PMF and transfusion dependence who achieved a durable, effective response with the combination of fedratinib and luspatercept.

\section{Case Report/Case Presentation}

The patient is a 66-year-old man who initially presented 8 years ago with splenomegaly and bicytopenia (hemoglobin [Hb] $10.3 \mathrm{~g} / \mathrm{dL}$, platelet count $128 \mathrm{~K} / \mu \mathrm{L}$ ). White blood cell count was normal at $7.52 \mathrm{~K} / \mu \mathrm{L}$. Peripheral smear showed the presence of teardrop cells, nucleated red cells, and rare blasts. There was no palpable lymphadenopathy on physical exam; however, the spleen was palpable at $12 \mathrm{~cm}$ below the costal margin. A computed tomography scan of the abdomen revealed a markedly enlarged spleen measuring $23 \mathrm{~cm}$ in length. Given the presence of asymptomatic bicytopenia and splenomegaly, a bone marrow aspiration and biopsy were performed due to clinical suspicion for a primary hematological neoplasm. The bone marrow biopsy showed a hypercellular bone marrow with marked reticulin fibrosis $(3+)$, trilineage maturation with blood leukoerythroblastosis (shown in Fig. 1). These findings were consistent with a diagnosis of PMF in fibrotic stage. Molecular studies revealed the presence of $J A K 2 V 617 F$ mutation by polymerase chain reaction testing. Fluorescent in situ hybridization testing revealed no evidence of deletion 5q. Risk stratification was completed utilizing the IPSS, and the patient's disease was deemed low-risk (0 points). As the patient was asymptomatic at the time, the decision was to implement observation.

Over the subsequent 3 years after initial presentation, the patient was being monitored periodically through office visits and laboratory evaluation. He was noted to have gradual worsening of anemia which prompted a repeat bone marrow aspiration and biopsy. It showed stable hypercellularity and reticulin fibrosis without increased myeloid blasts. Cytogenetic analysis revealed the presence of deletion in the long arm of chromosome 20 in $80.2 \%$ interphase nuclei by fluorescent in situ hybridization analysis. Risk stratification using the Dynamic IPSS-Plus placed the patient's disease in the INT-2 risk group [6]. The decision at the time was to initiate ruxolitinib. He initially had a $50 \%$ reduction in splenomegaly; however, he experienced worsening anemia requiring transfusion dependence, which led to dose reductions with ensuant recurrent splenomegaly to $20 \mathrm{~cm}$ on imaging. The option of bone marrow transplantation was discussed but deferred by the patient.

The patient was then enrolled in a phase III clinical trial evaluating pacritinib in patients with PMF (NCT03165734). This resulted in stabilization of the patient's splenomegaly without

\section{Karger'}




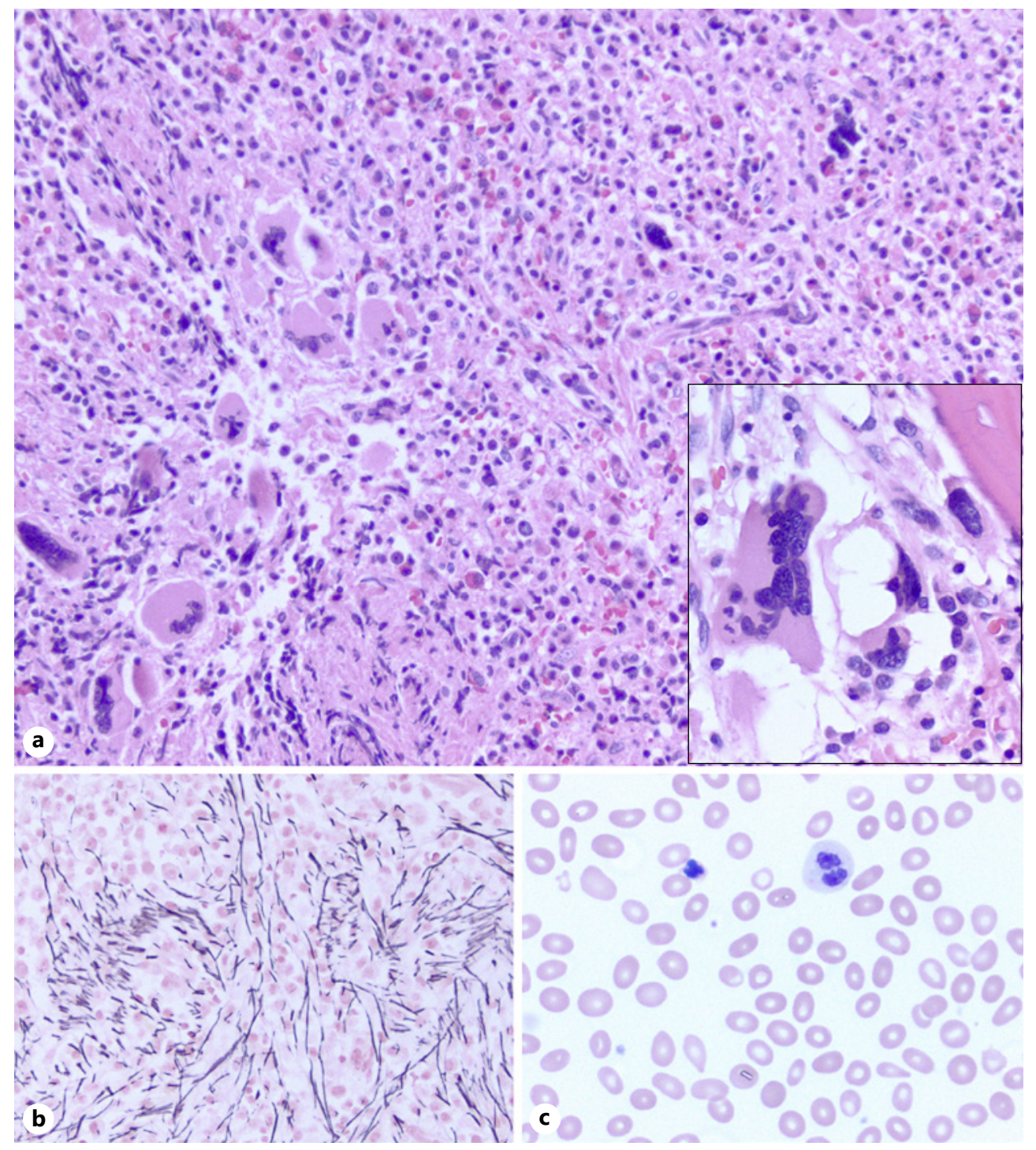

Fig. 1. Initial bone marrow biopsy and corresponding blood smear. a The biopsy shows $100 \%$ cellular marrow with trilineage hematopoiesis. Megakaryocytes are increased in concentration and clustered, with occasional forms abnormally located adjacent to the bone or showing increased number of nuclei or nuclear hypolobation (a, inset). b Reticulin staining shows MF-2 fibrosis, with patchy sparing. c The blood smear shows moderate anisopoikilocytosis with teardrops, rare nucleated RBCs, and occasional dysplastic neutrophils with hypogranular cytoplasm.

improvement in transfusion requirements. The drug was well tolerated; however, he was ultimately taken off study. The patient's transfusion requirement worsened thereafter. Nextgeneration sequencing on peripheral blood showed JAK2 as the sole molecular derangement. He required four units of packed red blood cell in the 2 months after being taken off trial and so was started on darbepoetin alfa. Baseline erythropoietin level was $50.9 \mathrm{U} / \mathrm{L}$. Transfusion requirements did not improve with the use of erythrocyte stimulating agents. He also developed symptomatic splenomegaly, with imaging revealing a spleen size of $26 \mathrm{~cm}$.

Given the FDA-approval of fedratinib in subsequent months, the patient was started on fedratinib $300 \mathrm{mg}$ daily due to thrombocytopenia, anemia, and renal function, while continuing darbepoetin alfa bimonthly. This dose was well tolerated and 2 months later, was increased 


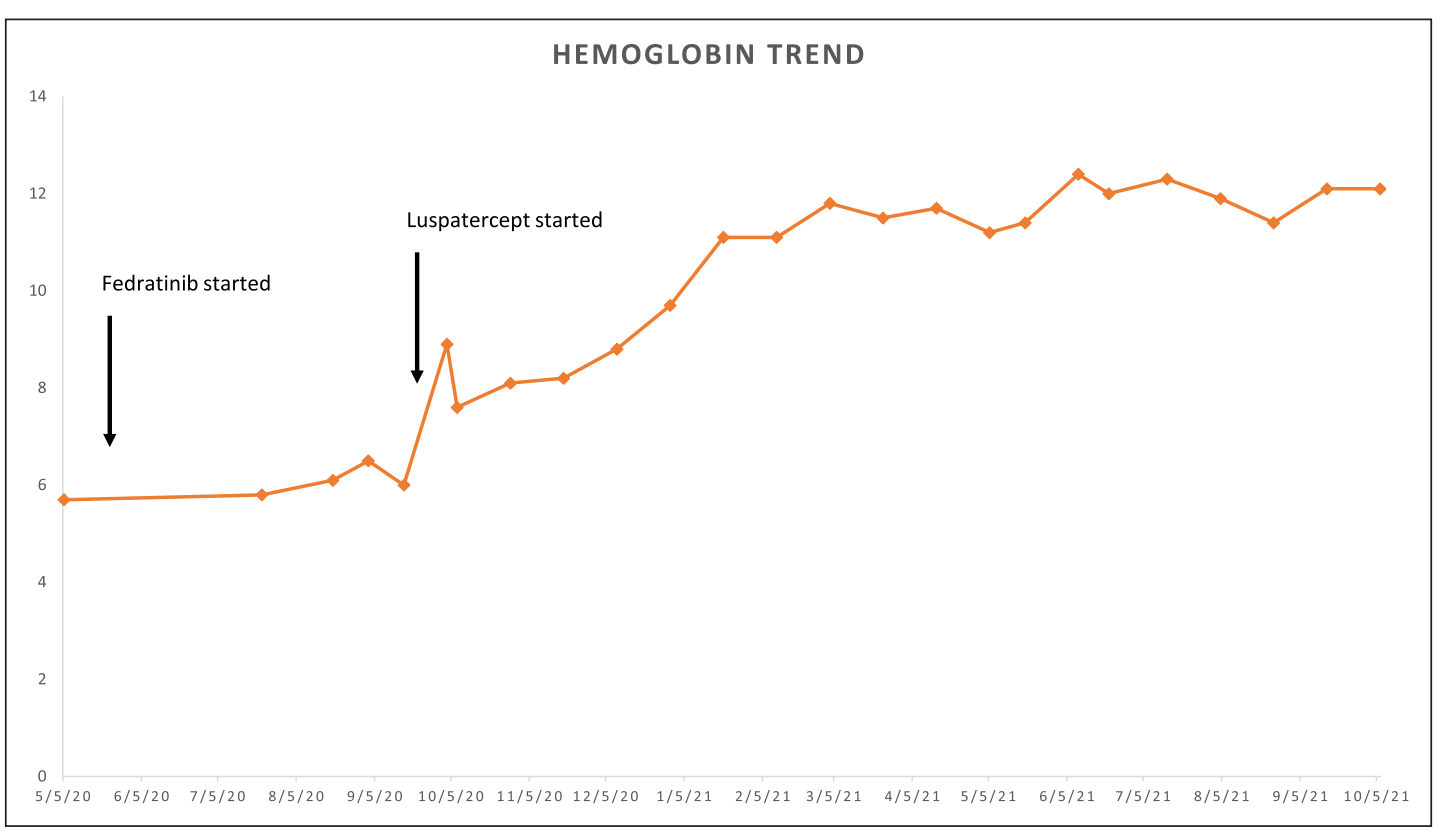

Fig. 2. Hb trend from addition of fedratinib and luspatercept to present day.

to $400 \mathrm{mg}$ daily. Periodic thiamine measurements were all normal, and no adverse events were experienced. Repeat imaging showed a 54\% reduction in the spleen size from $26 \mathrm{~cm}$ to $12 \mathrm{~cm}$. Despite this improvement, the patient continued to be transfusion dependent. Four months after the initiation of fedratinib, subcutaneous luspatercept $1 \mathrm{mg} / \mathrm{kg}$ every 2 weeks was added to the patient's therapy. Remarkably, the patient became transfusion independent after the first dose of luspatercept with his $\mathrm{Hb}$ gradually improving from a baseline of $6.0 \mathrm{~g} / \mathrm{dL}$ to $8.9 \mathrm{~g} / \mathrm{dL} 18$ days after the first dose of luspatercept. Timeline of $\mathrm{Hb}$ trend is shown in Figure 2. The patient also reported improvement in his appetite and had gained weight.

After 4 months of receiving luspatercept at its lowest dose and continuing fedratinib, the patient's $\mathrm{Hb}$ stabilized around $11.5 \mathrm{~g} / \mathrm{dL}$ and luspatercept was intermittently held throughout this period due to a Hb above $11.5 \mathrm{~g} / \mathrm{dL}$ (highest $12.3 \mathrm{~g} / \mathrm{dL}$ ). He continues to be on fedratinib $400 \mathrm{mg}$ daily without the need for luspatercept and remains transfusion independent 13 months since the initiation of luspatercept. The patient has had significant improvement in his performance status and is now back to working full time.

\section{Discussion}

Here, we present the case of a patient with PMF who had an exceptional response to combination fedratinib and luspatercept. As most JAK inhibitors are associated with the development of anemia, we believe that this combination is a viable option for patients with MF. Based upon the revised International Working Group-Myeloproliferative Neoplasms Research and Treatment criteria [7], our patient met criteria for both anemia response (becoming transfusion-dependent to transfusion-independent) in addition to spleen response (computed tomography showing $\geq 35 \%$ spleen volume reduction) with this regimen. He fulfilled the International Working Group-Myeloproliferative Neoplasms Research and Treatment and European LeukemiaNet (ELN) peripheral blood criteria for partial remission $(\mathrm{Hb}>10 \mathrm{~g} / \mathrm{dL}$ and < upper normal limit; absolute neutrophil count $>1,000 / \mathrm{mm}^{3}$; platelet count $>100 \mathrm{~K}$,

\section{Karger's}


immature cells $<2 \%$ ). No bone marrow biopsy sampling is available to determine if the patient has achieved a complete response.

In 2019, the FDA approved fedratinib for use in adults with INT-2 or high-risk PMF who are either treatment-naïve, resistant, or intolerant to ruxolitinib [8]. Fedratinib is a JAK2 selective inhibitor which showed significant symptom improvement and reduction in spleen size in the placebo-controlled, randomized phase II and III clinical trials, JAKARTA-1, and JAKARTA-2 $[9,10]$. In both studies, permanent discontinuation of fedratinib due to cytopenias was infrequent (about $2-3 \%$ of patients), suggesting milder suppression of hematopoiesis and an improved tolerability profile.

There are several mechanisms which may explain fedratinib's milder effect on anemia. It is thought that anemia in patients with MF is driven in part by excess production of hepcidin, a peptide hormone produced by hepatocytes which serve as a key regulatory of iron homeostasis [11]. Hepcidin reduces duodenal iron absorption and increases iron sequestration in monocytes and macrophages. Excess levels also reduce iron availability and restrict erythropoiesis [12]. Inflammation increases synthesis of hepcidin through activating the JAK2-STAT pathway [13]. Hepatic hepcidin production also occurs in response to aberrant cytokinedriven signaling via activin receptor type 1 , a member of the transforming growth factor- $\beta$ (TGF- 31 ) superfamily of receptors [14]. Fedratinib has dual activity in inhibiting both JAK2/ STAT3 signaling and TGF- $\beta 1 /$ Smad and non-Smad pathways, and this dual pathway inhibition leads to decreased hepcidin production in patients with MF $[15,16]$. Additionally, inhibition of SMAD signaling enables erythroid maturation by means of late-stage erythroblast differentiation thereby improving anemia. Fedratinib has also been shown to exert off-target inhibitory activity against bromodomain-containing protein 4 [4]. The combination of JAK/ STAT and bromodomain-containing protein 4 inhibition has been shown in animal models to synergistically block inflammatory cytokine production, attenuating disease burden and fibrosis in patients with MF and further reducing production of hepcidin [17].

While JAK2 inhibitors in PMF can lead to improved outcomes in splenomegaly and symptom burden, it may also worsen anemia, as previously noted. In patients who are transfusion independent and have low serum erythropoietin $(<125 \mathrm{U} / \mathrm{L})$, erythroid stimulating agents can be used however less than half of patients will respond and for the patients who do respond, the effect is not durable [18].

Luspatercept is a recombinant fusion protein which binds select TGF-beta superfamily ligands and blocks the Smad2/3 signaling pathway to enhance late-stage erythropoiesis $[19,20]$. In the MEDALIST trial [21], use of this agentled 38-53\% of patients with low-risk myelodysplastic syndromes and who were transfusion dependent and either refractory or intolerant to erythropoiesis-stimulating to achieve transfusion independence with improvement in erythrocyte count. TGF- $\beta 1$ ligand traps such as luspatercept and sotatercept have only recently started to gain use in PMF. In 2020, Bose et al. [22] published results from a phase II, single-institution study evaluating effects of sotatercept on patients with PMF or myeloproliferative neoplasm-associated MF who were anemic or transfusion dependent. They found a response rate of $29 \%$ when used as monotherapy or in conjunction with ruxolitinib. Data have been limited to patients who have secondary MF, and there exist little data on the effect of these agents in patients with PMF.

It remains unclear whether the patient's sudden response was solely a result of introducing luspatercept or if the addition of luspatercept to a JAK inhibitor resulted in an additive or synergistic effect of one or both medications. Additionally, next-generation sequencing continued to show JAK2 as the sole molecular abnormality. This may in part explain the favorable outcome that our patient has had. While the combination of fedratinib and luspatercept was successful for our patient in controlling symptoms, helped him to achieve transfusion-independence and improve his quality of life, there is no evidence for whether these two agents used in conjunction have any influence over disease progression and survival.

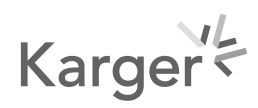


Notably, our patient had a durable response to luspatercept; he continues to be transfusionindependent for approximately 13 months. Randomized trials are needed to confirm if this observation can be seen in other patients.

\section{Conclusion}

MF-related anemia poses a challenge in the management of PMF. It has been associated with inferior quality of life and poor prognosis. There is an unmet need for effective treatment that led to achieving transfusion-independence. We present the case of a 66-year-old man with Dynamic IPSS-Plus INT-2 PMF who achieved transfusion-independence with the use of combination fedratinib and luspatercept. There are several ongoing clinical trials assessing ruxolitinib and JAK2 inhibitor-based combination therapy for the treatment of PMF [23]. In particular, the combination of fedratinib and luspatercept is a potentially promising therapy for anemia and reduction of symptom burden in patients with transfusion-dependent INT-2 MF. Currently, there are three clinical trials aiming to evaluate the safety and efficacy of luspatercept used either as monotherapy (NCT03194542) or in combination with a JAK2 inhibitor (NCT04717414 and NCT03755518) for the treatment of MF-associated anemia. We eagerly await the results of these ongoing trials.

\section{Statement of Ethics}

This study protocol was reviewed, and the need for approval was waived by the Internal Review Board Administration at Rush University Medical Center. The committee has deemed that this study does not constitute research or human subjects' research and therefore has granted an exemption from requiring ethics approval. Written informed consent was obtained from the patient for publication of the details of their medical case and any accompanying images.

\section{Conflict of Interest Statement}

The authors have no conflicts of interest to disclose.

\section{Funding Sources}

No funding was obtained for the preparation of this manuscript.

\section{Author Contributions}

Authors Yun, Alrifai, Miller, and Shammo contributed to the manuscript of the project. Author Miller provided the images and description in Figure 1.

\section{Data Availability Statement}

Data sharing is not applicable to this article as no new data were created or analyzed in this study. Additional patient information is not publicly available on legal and ethical grounds in order to protect patient confidentiality.

\section{Karger'}




\section{References}

1 Tefferi A. Primary myelofibrosis: 2021 update on diagnosis, risk-stratification and management. Am J Hematol. 2021 Jan;96(1):145-62.

2 Harrison CN, Schaap N, Mesa RA. Management of myelofibrosis after ruxolitinib failure. Ann Hematol. 2020 Jun;99(6):1177-91.

3 Tefferi A, Guglielmelli P, Pardanani A, Vannucchi AM. Myelofibrosis treatment algorithm 2018. Blood Cancer J. 2018 Jul;8(8):72.

4 Talpaz M, Kiladjian JJ. Fedratinib, a newly approved treatment for patients with myeloproliferative neoplasmassociated myelofibrosis. Leukemia. 2021 Jan;35(1):1-17.

5 Lee SE. Disease modifying agents of myeloproliferative neoplasms: a review. Blood Res. 2021 Apr;56(S1): S26-33.

6 Gangat N, Caramazza D, Vaidya R, George G, Begna K, Schwager S, et al. DIPSS plus: a refined Dynamic International Prognostic Scoring System for primary myelofibrosis that incorporates prognostic information from karyotype, platelet count, and transfusion status. J Clin Oncol. 2011 Feb;29(4):392-7.

7 Tefferi A, Cervantes F, Mesa R, Passamonti F, Verstovsek S, Vannucchi AM, et al. Revised response criteria for myelofibrosis: International Working Group-Myeloproliferative Neoplasms Research and Treatment (IWGMRT) and European LeukemiaNet (ELN) consensus report. Blood. 2013 Aug;122(8):1395-8.

8 Mullally A, Hood J, Harrison C, Mesa R. Fedratinib in myelofibrosis. Blood Adv. 2020 Apr;4(8):1792-800.

9 Pardanani A, Harrison C, Cortes JE, Cervantes F, Mesa RA, Milligan D, et al. Safety and efficacy of fedratinib in patients with primary or secondary myelofibrosis: a randomized clinical trial. JAMA Oncol. 2015 Aug;1(5): 643-51.

10 Harrison CN, Schaap N, Vannucchi AM, Kiladjian JJ, Tiu RV, Zachee P, et al. Janus kinase-2 inhibitor fedratinib in patients with myelofibrosis previously treated with ruxolitinib (JAKARTA-2): a single-arm, open-label, nonrandomised, phase 2, multicentre study. Lancet Haematol. 2017 Jul;4(7):e317-24.

11 Zhou A, Allen MJ, Fulbright M, Fowles JS, Fisher D, Oh ST. Hepcidin is elevated in primary and secondary myelofibrosis and correlates with IL- 6 and IL-2R $\alpha$ but is high in patients treated with ruxolitinib. Blood. 2018 Nov; 132(S1):1760.

12 Oh ST, Talpaz M, Gerds AT, Gupta V, Verstovsek S, Mesa R, et al. ACVR1/JAK1/JAK2 inhibitor momelotinib reverses transfusion dependency and suppresses hepcidin in myelofibrosis phase 2 trial. Blood Adv. 2020 Sep; 4(18):4282-91.

13 Fung E, Nemeth E. Manipulation of the hepcidin pathway for therapeutic purposes. Haematologica. 2013 Nov; 98(11):1667-76.

14 Asshoff M, Petzer V, Warr MR, Haschka D, Tymoszuk P, Demetz E, et al. Momelotinib inhibits ACVR1/ALK2, decreases hepcidin production, and ameliorates anemia of chronic disease in rodents. Blood. 2017 Mar; 129(13):1823-30.

15 Ruan H, Luan J, Gao S, Li S, Jiang Q, Liu R, et al. Fedratinib attenuates bleomycin-induced pulmonary fibrosis via the JAK2/STAT3 and TGF- $\beta 1$ signaling pathway. Molecules. 2021 Jul;26(15):4491.

16 Mleczko-Sanecka K, Casanovas G, Ragab A, Breitkopf K, Müller A, Boutros M, et al. SMAD7 controls iron metabolism as a potent inhibitor of hepcidin expression. Blood. 2010 Apr;115(13):2657-65.

17 Katsarou A, Pantopoulos K. Hepcidin therapeutics. Pharmaceuticals. 2018 Nov;11(4):127.

18 Naymagon L, Mascarenhas J. Myelofibrosis-related anemia: current and emerging therapeutic strategies. Hemasphere. 2017 Dec;1(1):e1.

19 Fenaux P, Kiladjian JJ, Platzbecker U. Luspatercept for the treatment of anemia in myelodysplastic syndromes and primary myelofibrosis. Blood. 2019 Feb;133(8):790-4.

20 Kubasch AS, Fenaux P, Platzbecker U. Development of luspatercept to treat ineffective erythropoiesis. Blood Adv. 2021 Mar;5(5):1565-75.

21 Fenaux P, Platzbecker U, Mufti GJ, Guillermo GM, Buckstein R, Santini V, et al. Luspatercept in patients with lower-risk myelodysplastic syndromes. N Engl J Med. 2020 Jan;382(2):140-51.

22 Bose P, Pemmaraju N, Masarova L, Bledsoe SD, Daver N, Jabbour E, et al. Sotatercept (ACE-011) for anemia of myelofibrosis: a phase 2 study. Blood. 2020 Nov;136(S1):10-1.

23 Bose P, Verstovsek S. JAK inhibition for the treatment of myelofibrosis: limitations and future perspectives. Hemasphere. 2020 Aug;4(4):e424. 